\title{
MANEJO INTERNO DE RESÍDUOS BIOLÓGICOS E PERFUROCORTANTES EM INSTITUIÇÃO DE ENSINO SUPERIOR
}

\author{
Tatiane Bonametti Veiga ${ }^{1}$ \\ Adriana Aparecida Mendes ${ }^{2}$ \\ Angela Maria Magosso Takayanagui ${ }^{3}$
}

\begin{abstract}
RESUMO
As Instituições de Ensino Superior são responsáveis por grandes avanços científicos em diversas áreas do conhecimento. Contudo, muitas de suas atividades e experimentos resultam na geração de diferentes tipos de resíduos, muitos deles perigosos. Este estudo teve como objetivo levantar aspectos do manejo interno dos resíduos biológicos e perfurocortantes gerados no Campus da Universidade de São Paulo de Ribeirão Preto. Os dados revelaram que em $87,4 \%$ dos locais participantes da pesquisa havia a geração de algum tipo de resíduo de serviço de saúde. Em relação às fases de manejo interno foi verificado que procedimentos como a segregação na fonte, o acondicionamento em recipientes compatíveis com o tipo de resíduo e a identificação das embalagens nos laboratórios/serviços estavam adequados, em sua maioria, à legislação brasileira; porém, dados como o acondicionamento de resíduos perfurocortantes em sacos plásticos em 5,4\% dos locais que indicaram gerar esse tipo de resíduo, demonstram a inadequação na utilização de alguns procedimentos. Os achados revelaram a necessidade da implantação de um Plano de Gerenciamento Integrado de Resíduos no Campus, além de manter um cronograma contínuo de educação ambiental, a fim de contribuir para o gerenciamento ambiental e ter potencialidade para ser utilizado, também, como referência em outras instituições similares.
\end{abstract}

PALAVRAS-CHAVE: Instituições de Ensino Superior, gerenciamento de resíduos, resíduos de serviços de saúde.

\section{INTERNAL MANAGEMENT OF BIOLOGICAL AND SHARPS WASTE IN HIGHER EDUCATION INSTITUTION}

\section{ABSTRACT}

\footnotetext{
${ }^{1}$ Doutora em Ciências, Programa de Pós-graduação em Enfermagem em Saúde Pública, Universidade de São Paulo - USP. Engenheira Civil. tati.veiga@yahoo.com.br.

${ }^{2}$ Doutora em Ciências, Programa de Pós-graduação em Enfermagem em Saúde Pública, Universidade de São Paulo - USP. Enfermeira. adrianaapmendes@yahoo.com.br.

${ }_{3}^{3}$ Doutora em Enfermagem. Universidade de São Paulo - USP. Professora Associada da Escola de Enfermagem de Ribeirão Preto (EERP/USP). amtakay@eerp.usp.br.
} 
da Alta Paulista

The Higher Education Institutions are responsible for major scientific advances in many areas of knowledge. However, many of their activities and experiments result in the generation of different types of waste, many of them dangerous. This study aimed to lift aspects of internal handling of biological and sharps waste generated on the Campus of the University of São Paulo at Ribeirão Preto. Data revealed that in $87.4 \%$ of the researched places produced some types of medical waste. Related to the steps of internal management was verified if procedures such as segregation at source, packaging in containers compatible with the type of waste, and identification of packaging in the laboratories and services were appropriated to the Brazilian legislation in most situations. However, the data regarding the packaging sharps waste in plastic bags demonstrated the inadequacy in the use of some procedures in 5.4\% of the places, which generated such wastes. Findings showed need for the implementation of an Integrated Management Plan for Waste of the Campus, besides maintaining an ongoing schedule for in-service environmental education in order to contribute to the environmental management and have also the potentiality to be used as reference in other similar institutions.

KEY-WORDS: Higher Education Institutions, waste management, medical waste.

\section{GESTIÓN INTERNA DE RESIDUOS BIOLÓGICOS Y PUNZOCORTANTES EN INSTITUCIÓN DE ENSEÑANZA SUPERIOR}

\begin{abstract}
RESUMEN
Instituciones de Enseñanza Superior son responsables por grandes avances científicos en muchas áreas del conocimiento. Sin embargo, muchas de sus actividades y experimentos resultan en la generación de diferentes tipos de residuos, muchos de ellos peligrosos. Este estudio objetivó abordar los aspectos de la gestión interna de los residuos biológicos y punzocortantes generados en el Campus de la Universidad de São Paulo de Ribeirão Preto. Los datos mostraron que en el $87,4 \%$ de los sitios participantes de la investigación producía algún tipo de residuo sanitario. En relación a las fases de la gestión interna se verificó que los procedimientos como la segregación en la fuente, el embalaje en recipientes compatibles con el tipo de residuo y la identificación de los embalajes en los laboratorios/servicios eran adecuadas a la legislación brasileña en la mayoría de las situaciones. Sin embargo, datos como el embalaje de residuos punzocortantes en bolsas de plástico en el 5,4\% de los sitios que indicaron generar este tipo de residuos, demostraran el uso inadecuado de algunos procedimientos. Los hallazgos revelan la necesidad de implementar un Plan de Manejo Integrado de Residuos en el Campus, y mantener una programación permanente de educación ambiental con el fin de contribuir para la gestión ambiental y tener el potencial de ser utilizado también como referencia en otras instituciones.
\end{abstract}

PALABRAS-CLAVE: Instituciones de Enseñanza Superior. Administración de Residuos. Residuos Sanitarios. 


\section{INTRODUÇÃO}

A preocupação com a excelência do desenvolvimento ambiental e suas interrelações com as dimensões sociais e econômicas não pode mais ficar restrita somente ao setor produtivo. É necessário o envolvimento da sociedade e do poder público para discussão e formulação de novas políticas públicas para atender as demandas que surgem frente às mudanças que vêm ocorrendo nas últimas décadas.

As Instituições de Ensino Superior (IES) apresentam um papel fundamental nesse contexto. A preocupação com essas novas mudanças faz com que ocorra um maior envolvimento dos alunos, professores, funcionários e da comunidade acadêmica nas discussões pela busca de melhores formas de controle que visem minimizar os impactos causados por atividades desenvolvidas nessas instituições.

As IES conscientes da sua responsabilidade social e ambiental começaram, nos últimos anos, a promover várias mudanças a fim de: obter melhores índices referentes ao consumo de energia, de água e de materiais; estabelecer critérios mais rígidos de controle para adequação às legislações nacionais e locais; gerenciar seus recursos para minimizar o desperdício; intensificar os projetos de formação e treinamento de seus funcionários, reduzindo, assim, os riscos de incorrer em penalidades ou gerar passivos ambientais, intensificando as práticas responsáveis e promovendo a melhoria na imagem externa da instituição; além de gerar novas oportunidades de pesquisa (GOMES, 2010).

É possível perceber que várias ações descritas anteriormente estão, direta ou indiretamente, relacionadas a um problema que atinge não somente as instituições de ensino, bem como toda a sociedade. Dessa forma, uma das áreas consideradas como prioritárias, que vem causando grande preocupação diz respeito à gestão e gerenciamento de resíduos sólidos urbanos (RSU).

Entre esses resíduos destacam-se os resíduos de serviços de saúde (RSS), que, embora representem uma pequena porcentagem da produção total de RSU (TAKAYANAGUI, 2005), são alvo de grande preocupação devido a sua composição e seu potencial de periculosidade (PUGLIESI; GIL; SCHALCH, 2009), oferecendo risco à saúde e ao ambiente, por apresentarem características como: 
inflamabilidade, corrosividade, reatividade, toxicidade, patogenicidade, carcinogenicidade, teratogenicidade e mutagenicidade (BRASIL, 2010).

Os resíduos quando não manejados adequadamente geram danos à saúde e ao ambiente, podendo ser responsáveis por graves acidentes. As consequências podem ser ainda mais sérias, tornando-se extensiva a comunidade em geral, quando causam poluição do ar, água e solo, e intensificam a proliferação de insetos e roedores (MOTA, 1997).

A diversidade de resíduos gerados em muitas IES impõe aos seus administradores a necessidade de adotar medidas que visem a sua minimização e controle. Uma das medidas fundamentais nesse processo é adotar um modelo de gestão de resíduos adequado às necessidades específicas para cada situação, a fim de realizar seu gerenciamento com eficiência. Segundo Silva (2011), a falta de um programa eficiente de gestão de resíduos pode provocar a mistura dos RSS com os RSU colocando em risco toda a população de uma determinada região.

A preocupação com a questão ambiental, social e econômica faz do gerenciamento de resíduos uma ferramenta importante no controle e na minimização do uso de recursos naturais, assim como, na promoção da saúde e na preservação e conservação do meio ambiente (BRASIL, 2006). Gerenciar os resíduos de forma integrada corresponde em articular ações normativas, operacionais, financeiras e de planejamento, fundamentadas em critérios sanitários, ambientais e econômicos, empregando técnicas e tecnologias compatíveis com a realidade de cada local que busquem sempre reduzir, reaproveitar e reutilizar os resíduos gerados (SCHALCH; LEITE; CASTRO, 2003).

Para realizar um gerenciamento de resíduos adequado, devem ser consideradas algumas diretrizes como conhecer a realidade local $e$ as particularidades regionais, delimitando o problema de forma a compreender o fluxo dos resíduos; conhecer suas dimensões, no nível social, econômico e cultural; realizar um planejamento detalhado e consciente do gerenciamento de forma integrada a outros projetos e ter a participação da população (FERNANDEZ, 2008).

Segundo o Instituto de Pesquisas Tecnológicas do Estado de São Paulo (2000), conhecer as características dos resíduos gerados é fundamental para adotar medidas adequadas no seu gerenciamento, dessa forma a realização de um 
diagnóstico atualizado é essencial para auxiliar na elaboração e acompanhamento de um modelo eficiente de gerenciamento de resíduos. A importância desse diagnóstico é ressaltada como uma das primeiras fases no manual da Agência Nacional de Vigilância Sanitária (Anvisa) para a elaboração do Plano de Gerenciamento de Resíduos de Serviços de Saúde (PGRSS) (BRASIL, 2006).

O presente estudo teve como objetivo identificar a forma de manejo interno (segregação, acondicionamento, identificação, armazenamento, coleta, transporte e tratamento) dos resíduos biológicos e perfurocortantes gerados no Campus de Ribeirão Preto da Universidade de São Paulo (USP).

\section{MATERIAL E MÉTODOS}

O local de estudo consiste no Campus da USP de Ribeirão Preto, cidade situada na região nordeste do estado de São Paulo. Segundo estimativa do Instituto Brasileiro de Geografia e Estatística (IBGE), o município possui uma população estimada em mais de 650 mil habitantes (IBGE, 2015).

Esse Campus possui uma circulação diária de aproximadamente 20 mil pessoas, entre docentes, discentes, funcionários e pacientes do Hospital das Clínicas da Faculdade de Medicina de Ribeirão Preto da Universidade de São Paulo (HCFMRP/USP). A área do Campus corresponde a 585,89 ha, localizando-se em antiga fazenda de café, nos limites da Zona Oeste da cidade.

Compreende nove Unidades de Ensino, Pesquisa e Extensão, além do Hospital das Clínicas, Hemocentro, Restaurante Universitário e vários outros serviços de apoio e extensão à comunidade. A Instituição encontra-se em franca fase de expansão, com contínuos projetos de construção ou ampliação das instalações de suas Unidades de Ensino e Serviços.

Frente a essa expansão foi realizado este estudo de caráter descritivo e exploratório, baseado na elaboração de um diagnóstico da situação do manejo interno dos resíduos sólidos, com foco nos resíduos biológicos e perfurocortantes no Campus da USP de Ribeirão Preto. 
Para realizar esta investigação foi elaborado um questionário com perguntas semiestruturadas sobre o manejo interno dos diferentes tipos de resíduos gerados nos laboratórios/serviços no Campus da USP de Ribeirão Preto. Nessa construção foram seguidas as normas vigentes no Brasil, que definem a classificação dos resíduos e indicam os cuidados que devem ser adotados no manejo nas diferentes fases do seu gerenciamento com base nos seguintes documentos: NBR 10.004/04 (ABNT, 2004), as resoluções $n^{\circ} 358 / 05$, do Conselho Nacional do Meio Ambiente (Conama) e a RDC n`306/04 da Anvisa (BRASIL, 2004, 2005), além de considerar os princípios presentes na Lei da Política Nacional de Resíduos Sólidos (BRASIL, 2010).

Foram convidados para esta investigação 299 sujeitos responsáveis pelos laboratórios/serviços ou algum técnico/pesquisador por eles indicados, que responderam a um questionário referente ao gerenciamento dos resíduos, tendo como parâmetro o seu local de trabalho.

Os dados foram organizados em planilhas de acordo com os itens do questionário referente à geração, categorizando-os segundo a classificação da RDC no 306/04 (BRASIL, 2004), destacando-se cada fase do manejo interno dos resíduos biológicos e perfurocortantes gerados no referido Campus.

Em cumprimento às normas da Comissão Nacional de Ética em Pesquisa, presentes na Resolução do Conselho Nacional de Saúde no 196/96 (BRASIL, 1996), o projeto foi aprovado pelo Comitê de Ética em Pesquisa da Escola de Enfermagem de Ribeirão Preto.

\section{RESULTADOS E DISCUSSÃO}

Dos questionários entregues aos responsáveis pelos laboratórios/serviços do Campus, 66,6\% foram devolvidos respondidos.

Os dados em relação aos tipos de resíduos gerados evidenciaram uma situação preocupante ao revelar que $65,3 \%$ dos sujeitos indicaram que em seus laboratórios/ serviços eram gerados resíduos biológicos e 69,4\% informaram gerar resíduos perfurocortantes. 
Em relação à segregação, os dados revelaram que era realizada, em sua maioria, no momento da geração, o que ocorreu em $84,7 \%$ no caso dos resíduos biológicos e em $82,7 \%$ dos resíduos perfurocortantes. Nesse tipo de segregação 0 próprio gerador realiza a separação dos resíduos, o que minimiza os riscos de acidentes. Contudo, foi possível observar que houve um índice considerável de sujeitos que informaram realizar a segregação posteriormente, como no caso resíduos perfurocortantes $(10,8 \%)$, não sendo essa uma prática adequada segundo a legislação vigente (BRASIL, 2004, 2005).

A segregação é o primeiro passo do manejo dos resíduos e deve ser realizada na própria fonte geradora. Essa fase é considerada fundamental por diferentes especialistas (ASKARIAN; MOTAZEDIAN; PALENIK, 2011; GÜNTHER, 2010) para permitir o cumprimento dos objetivos de um sistema eficiente e seguro de manuseio que consiste em separar os resíduos de acordo com sua classificação (BRASIL, 2006).

Segundo as respostas obtidas, foi possível observar que havia uma prática frequente de acondicionamento nos laboratórios que também segue orientações constantes nas normas e resoluções que regulamentam o acondicionamento dos resíduos de diferentes grupos.

Em relação aos resíduos biológicos, 72,2\% dos sujeitos indicaram que o seu acondicionamento era realizado em sacos plásticos brancos, estando de acordo com as normatizações da NBR 9.191/08 e RDC 306/04 (ABNT, 2008; BRASIL, 2004).

Quanto ao acondicionamento dos resíduos perfurocortantes, foi possível verificar que a maioria dos sujeitos $(72,8 \%)$ referiu utilizar caixas de papelão resistentes para o acondicionamento desses materiais e $11,6 \%$ dos sujeitos indicaram que utilizavam recipientes de plástico reforçado. Dessa forma, segundo os sujeitos participantes, o acondicionamento da maioria desses resíduos em seus laboratórios/serviços estava de acordo com as especificações da RDC oㅜ 306/04, que exige o acondicionamento de resíduos perfurocortantes em recipientes rígidos, resistentes à punctura, ruptura e vazamento (BRASIL, 2004). Contudo, 5,4\% dos sujeitos informaram que esses resíduos eram acondicionados em sacos plásticos, o que representa um dado preocupante por oferecer um o eminente de acidente às pessoas que têm contato com esses resíduos. 
$\mathrm{Na}$ Tabela 1 é apresentado um resumo das respostas referentes ao acondicionamento dos diferentes tipos de resíduos realizados nos laboratórios/serviços.

Tabela 1 - Distribuição dos resíduos biológicos e perfurocortantes gerados nos laboratórios/serviços do Campus da USP de Ribeirão Preto, segundo o seu modo de acondicionamento

\begin{tabular}{|c|c|c|c|c|c|c|}
\hline $\begin{array}{c}\text { Acondicionamento } \\
\text { (\%) }\end{array}$ & 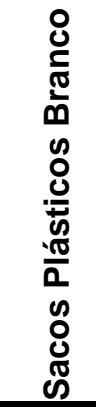 & 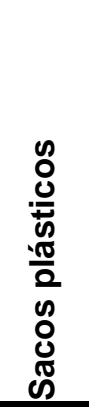 & 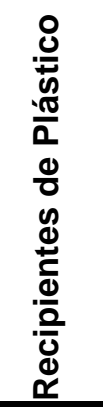 & $\begin{array}{l}\bar{\Phi} \\
\frac{0}{\pi} \\
\frac{0}{0} \\
\frac{1 \pi}{0} \\
\bar{d} \\
\frac{0}{\pi} \\
\frac{0}{0} \\
\mathbb{0} \\
\frac{0}{\pi} \\
0 \\
\end{array}$ & 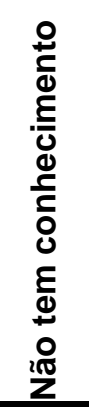 & 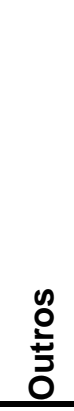 \\
\hline \multicolumn{7}{|l|}{ Grupo Resíduos } \\
\hline Biológico & 72,2 & - & 17,9 & 6,0 & 1,3 & 2,6 \\
\hline Perfurocortante & - & 5,4 & 11,6 & 72,8 & 6,1 & 4,1 \\
\hline
\end{tabular}

Somente $65,8 \%$ dos sujeitos informaram que era realizada a identificação das embalagens onde estavam armazenado os resíduos. Em relação ao armazenamento interno, $47,2 \%$ dos sujeitos referiram não existir um local exclusivo, 43,7\% afirmaram que seus laboratórios/serviços possuíam um local exclusivo para armazenamento interno dos resíduos e 9,1\% dos sujeitos não tinham desconhecimento a esse respeito.

Segundo as respostas dos sujeitos em relação à coleta interna, 50,8\% das pessoas que realizavam esse serviço eram funcionários da própria instituição, 5,2\% informaram não ter conhecimento sobre o responsável pela realização da coleta interna; porém, 36,7\% eram funcionários contratados de empresa privada e o restante da coleta era realizada por alunos ou outros técnicos da instituição $(7,3 \%)$, fator que dificulta o planejamento de treinamentos, supervisão e controle, devido à alta rotatividade desses funcionários/alunos.

Quanto ao transporte interno dos resíduos, foi averiguado que, em vários locais, o transporte interno não era realizado de uma única forma. Na maioria dos 
casos, o transporte interno era realizado manualmente $(63,3 \%)$ e somente em 6,5\% dos casos era utilizado carrinho com tampa para realizar o transporte, conforme as especificações da RDC n 306/04 (BRASIL, 2004).

Em relação ao tratamento interno, os sujeitos informaram que 64,3\% dos laboratórios/serviços do Campus da USP de Ribeirão Preto não realizam nenhum tipo de tratamento interno de seus resíduos, o que demonstra um campo a ser trabalhado na instituição, visto que o tratamento interno de alguns tipos de resíduos pode ser realizado no próprio local de sua geração segundo as normas de segurança vigentes para cada tipo de resíduo.

\section{CONCLUSÕES}

Os problemas relacionados ao gerenciamento de resíduos de serviços de saúde em Instituições de Ensino Superior não são somente físicos, químicos ou biológicos, mas envolvem mudanças comportamentais de toda a comunidade acadêmica, incluindo o setor administrativo. As mudanças de conduta ocorrem de maneira gradativa, requerem muito esforço e treinamento periódico para aumentar a conscientização de todos os envolvidos no processo de manejo dos resíduos, sendo primordial o apoio dos órgãos superiores das instituições durante todo esse processo (DE CONTO, 2010).

As universidades, como instituições responsáveis pela produção do conhecimento e pela formação de recursos humanos, não podem mais se isentar de sua responsabilidade ambiental, social e legal. Essas instituições são referência para a sociedade e devem empenhar seus esforços para buscar um desenvolvimento de forma sustentável, com programas efetivos de educação ambiental que busquem o equilíbrio entre proteção ambiental, crescimento econômico e equidade social, de forma a contribuir para a disseminação de novos conceitos e práticas.

Os dados levantados neste trabalho demonstram os avanços do referido Campus quanto à gestão dos resíduos biológicos e perfurocortantes gerados em suas delimitações nos últimos anos. Porém, o diagnóstico realizado ainda evidencia 
uma dificuldade dos geradores devido à falta de conhecimento quanto às técnicas mais adequadas para o gerenciamento de cada tipo de resíduo, frente à diversidade e quantidade de resíduos gerados em seus laboratórios/serviços. Outro fator que pode ser observado foi referente à falta de interação entre as pessoas envolvidas nas diferentes fases do manejo, destacando o fato de muitos funcionários serem terceirizados, com contratos temporários, o que dificulta o processo de treinamento contínuo e especializado.

A geração de resíduos em universidades é inerente ao desenvolvimento das atividades de ensino, pesquisa e extensão. Assim, visando continuar o processo de melhoria para a gestão de resíduos é necessário à implementação de um Plano de Gestão Integrado de Resíduos Sólidos (PGIRS) por uma equipe multidisciplinar, com a participação da administração, ressaltando a importância em se adotar uma padronização adequada no manejo dos resíduos gerados no Campus da Universidade de São Paulo de Ribeirão Preto, embasado nas legislações vigentes.

Outra questão que deve ser abordada consiste na continuidade da promoção de ações educativas, cursos e treinamentos, não somente para informar e treinar os funcionários e estudantes para a utilização de técnicas/procedimentos eficientes no manejo dos resíduos de diferentes grupos, mas, principalmente, por proporcionar a formação de cidadãos críticos, capazes de atuar com consciência, na busca pela minimização de técnicas que agridem o ambiente.

A partir da realização deste estudo, espera-se que outras Instituições de Ensino Superior, à luz de uma preocupação ambiental e social, possam utilizar esta investigação como parâmetro para a realização de novos estudos na área de gestão de resíduos.

\section{Agradecimentos}

Esta pesquisa teve 0 apoio financeiro do Conselho Nacional de Desenvolvimento Científico e Tecnológico (CNPq). 


\section{REFERÊNCIAS}

ASKARIAN, Mehrdad; MOTAZEDIAN, Nasrin; PALENIK, Charles John. Clinical laboratory waste management in Shiraz, Iran. Waste Management \& Research, v. 30, n.6, p. 631-634, 2011.

[ABNT] Associação Brasileira de Normas Técnicas. NBR 9.191: Sacos plásticos para acondicionamento de lixo - requisitos e métodos de ensaio. Rio de Janeiro: 2008. 10 p.

NBR 10.004: Resíduos sólidos - Classificação. Rio de Janeiro: 2004. 71 p.

BRASIL. Lei no 12.305, de 02 de agosto de 2010. Institui a Política Nacional de Resíduos Sólidos; altera a Lei no 9.605, de 12 de fevereiro de 1998; e dá outras providencias. Diário Oficial da União. Brasília, DF, 03 ago. 2010.

Ministério da Saúde. Manual de gerenciamento de resíduos de serviço de saúde. Brasília, DF: Ministério da Saúde, 2006. 182p.

Conselho Nacional do Meio Ambiente. Resolução $n^{\circ} 358$. Dispõe sobre o tratamento e a disposição final dos resíduos de saúde e dá outras providências. Diário Oficial da União. Brasília, DF, 04 maio 2005.

Agência Nacional de Vigilância Sanitária. Resolução RDC n 306, de 07 de dezembro de 2004. Dispõe sobre o regulamento técnico para o gerenciamento de resíduos de serviços de saúde. Diário Oficial da União. Brasília, DF, 10 dez. 2004.

Resolução no 196/96. Diretrizes e normas regulamentadoras sobre pesquisa envolvendo seres humanos. Conselho Nacional de Saúde. Diário Oficial da União. Brasília, DF, 10 out. 1996.

DE CONTO, Suzana Maria. Gestão de Resíduos em universidades: uma complexa relação que se estabelece entre heterogeneidade de resíduos, gestão acadêmica e mudanças comportamentais. In: DE CONTO, Suzana Maria. Gestão de Resíduos em Universidades. Caxias do Sul, RS: Educs, 2010. cap. 1, p. 17-32.

FERNANDEZ, Jaqueline Aparecida Bória. Diagnóstico e diretrizes para o gerenciamento de resíduos perigosos domiciliares: estudo de caso do município de São Carlos. 2008. 200 f. Tese (Doutorado em Ciências da Engenharia Ambiental) - Escola de Engenharia de São Carlos, Universidade de São Paulo, São Carlos, 2008.

GOMES, Luciana Paulo. A gestão de resíduos na Universidade do Vale do Rio dos Sinos (Unisinos) atendendo os requisitos da ISO 14001:2004. In: DE CONTO, Suzana Maria. Gestão de resíduos em universidades. Caxias do Sul. RS: EDUCS, 2010. cap. 3, p. 61-85.

GÜNTHER, Wanda Maria Risso et al. Elaboração de plano de gerenciamento de resíduos de serviço de saúde - PGRSS. São Paulo: USP, 2010.

[IBGE] Instituto Brasileiro de Geografia e Estatística. Pesquisa Populacional. Disponível em: http://cidades.ibge.gov.br/xtras/perfil.php?codmun=354340. Acesso em:09/04/2015.

[IPT] Instituto de Pesquisas Tecnológicas do Estado de São Paulo. Compromisso Empresarial para Reciclagem - CEMPRE. Lixo municipal: manual de gerenciamento integrado. 2 ed. São Paulo: IPT/CEMPRE, 2000. $370 \mathrm{p}$.

MOTA, Suetônio. Introdução à Engenharia Ambiental. Rio de Janeiro: ABES,1997. 292p.

SILVA, Cesar Aparecido et al. Diagnóstico dos resíduos sólidos de serviços de saúde gerados em um instituição do ensino superior. Engenharia Ambiental, Espírito Santo do Pinhal, v. 8, n. 4, p. 100117, out/dez. 2011. 
da Alta Paulista

PUGLIESI, Erika; GIL, Tatiana Novis Lopes; SCHALCH, Valdir. Caracterização qualitativa e quantitativa dos resíduos de serviço de saúde gerados em hospital de médio porte no município de São Carlos, SP. Revista Minerva, São Carlos, v. 6, n. 2, p. 213-217, maio/ago. 2009.

SCHALCH, Valdir; LEITE, Wellington Cyro Almeida; CASTRO, Marcus Cesar Avezum Alves. Prevenção da poluição, análise de ciclo de vida, redução, reutilização e reciclagem de resíduos sólidos. São Carlos: EESC/USP, 2003. 107 p.

TAKAYANAGUI, Angela Maria Magosso. Gerenciamento de resíduos de serviços de saúde. In: PHILIPPI JÚNIOR, A. Saneamento, saúde e ambiente: fundamentos para um desenvolvimento sustentável. São Paulo: Manole, 2005. cap. 9, p. 323-374. 